Trauma Berufskrankh 2014 · 16[Suppl 1]:16-20 DOI 10.1007/s10039-013-2028-4

Online publiziert: 14. November 2013

(c) Springer-Verlag Berlin Heidelberg 2013
P.-M. Hax

Klinik für Orthopädie und Unfallchirurgie, BG-Unfallklinik Duisburg

\section{Kommunikation im berufsgenossenschaftlichen Heilverfahren}

\author{
Besonderheiten der Arzt- \\ Patienten-Kommunikation bei Eintritt \\ des Versicherungsfalls
}

\section{Hintergrund}

\section{Nicht optimale Kommunikation}

„Grüße aus Babel - Patienten verstehen Ärzte oft nicht“ - war im September 2012 in der Ärztezeitung [23] zu lesen. Kommunikationsprobleme mit Ärzten können sich negativ auf den Behandlungserfolg auswirken und erhöhen den Bedarf an Beratung und Schlichtung im Gesundheitswesen [23]. Beim Erstkontakt werden einleitende Worte des Patienten durchschnittlich schon nach etwa $20 \mathrm{~s}$ vom Arzt unterbrochen [18]. In 50\% der Fälle gehen Ärzte während des Gesprächs mit dem Patienten Nebentätigkeiten nach. Die Beziehung ist meist asymmetrisch mit einem Gesprächsanteil des Arztes von bis zu 80\%. Häufig kommt es zu Unterbrechungen durch Telefonate oder Fragen von Mitarbeitern. Das hat zur Folge, dass der Patient nur etwa 50\% des Gesagten versteht und davon wiederum die Hälfte nach 30 min vergessen hat. Nur knapp 30\% der Ärzte nehmen den Wunsch der Patienten nach Information adäquat wahr. Ein großer Teil der Beschwerden kommt in der Regel gar nicht zur Sprache. Der Aufbau eines Vertrauensverhältnisses ist auf diese Weise kaum möglich.

\section{Optimierte Kommunikation}

\section{Vorteile}

Für den Patienten sind die Vorteile einer gelungenen Kommunikation bekannt: Er fühlt sich als Person wahrgenommen, respektiert und in der Obhut des Arztes aufgehoben; Vertrauen und Zufriedenheit wachsen, die Compliance wird gestärkt, die Mitarbeit nimmt zu. Eine positive Wirkung auf den Heilverlauf und den Heilerfolg ist wissenschaftlich belegt [7, 13, 27].

Auch für den Arzt hat eine gelungene Kommunikation nachweislich Vorteile, die sich gut zu dem Stichwort Burnoutprävention zusammenfassen lassen: Die subjektive Belastung durch die Krankheit des Patienten nimmt ab, die Stressbelastung durch den Beruf wird als geringer empfunden, die berufliche Zufriedenheit wächst, und die Neigung zu Depressionen und Ängsten nimmt ab [2, 10, 19].

\section{Charakteristika}

Ein zeitgemäßes Arzt-Patient-Verhältnis ist dadurch gekennzeichnet, dass der Arzt umdenken und sich auf den informierten Patienten einstellen muss. Der klassische Heilauftrag wird durch eine Kunden-Leistungserbringer-Konstellation ersetzt, das Vertrauensverhältnis durch ein Vertragsverhältnis [12]. Der Arzt hat sein Wissensmonopol verloren, der Patient will in Entscheidungen im Sinne einer partizipativen
Entscheidungsfindung einbezogen werden (,shared decision making“, [9]).

Anerkannte Merkmale einer gelungenen Kommunikation sind aktives Zuhören, das Zeigen von Interesse und regelmäßiges Nachfragen, Zusammenfassen und Erklären. Der Arzt sollte Sicherheit und emotionale Wertschätzung vermitteln. Dabei muss man sich dessen bewusst sein, dass Kommunikation nur zu einem sehr geringen Anteil von unter 10\% durch verbale Signale wirkt, zu knapp 40\% durch vokale Signale wie Stimme, Lautstärke, Tonfall und zu über 50\% durch nichtverbale Signale wie Körperhaltung, Blick, Handbewegungen und Mimik. Kaum eine andere Fähigkeit schärft die ärztliche Urteilskraft mehr als Empathie [15]. Sachzwänge der apparativen Medizin, Bürokratisierung der Betriebsabläufe, Zersplitterung der Verantwortung in multiprofessionellen Teams und das vordringende Kosten-Nutzen-Denken behindern den Arzt allerdings immer stärker in der Erfüllung seines eigentlichen Auftrags: sich dem individuellen Patienten mit voller Aufmerksamkeit zu widmen [4].

\section{Bedeutung der Arzt-Patient- Kommunikation für Orthopädie und Unfallchirurgie}

Gerade im Gebiet der Orthopädie und Unfallchirurgie gibt es mittlerweile zahlreiche wichtige Gründe, sich mit dem 
Tab. 1 Rahmenbedingungen der Kommunikation in der Unfallchirurgie

\begin{tabular}{|c|c|}
\hline \multirow{4}{*}{$\begin{array}{l}\text { Auf Seiten des } \\
\text { Patienten }\end{array}$} & Unvorbereitet \\
\hline & Plötzlich vor neuer Ausgangslage stehend (Situation, Wissen, Erwartungen) \\
\hline & Schmerz \\
\hline & $\begin{array}{l}\text { Emotionale Belastung (Enttäuschung, Trauer, Wut, Sorgen, Zukunftsangst, } \\
\text { Reizüberflutung) }\end{array}$ \\
\hline \multirow[t]{5}{*}{$\begin{array}{l}\text { Auf Seiten des } \\
\text { Arztes }\end{array}$} & $\begin{array}{l}\text { Zwang zu schnellen Entscheidungen (Dringlichkeit der Versorgung, Häufung } \\
\text { von Notfallpatienten) }\end{array}$ \\
\hline & Häufig suboptimale äußere Bedingungen \\
\hline & Personelle Situation im Bereitschaftsdienst (quantitativ und qualitativ) \\
\hline & Bandbreite der Kommunikationsmöglichkeiten häufig begrenzt \\
\hline & $\begin{array}{l}\text { Breite Facette von Kommunikationswerkzeugen gefordert (Kinder/Eltern, } \\
\text { Senioren, Migranten) }\end{array}$ \\
\hline
\end{tabular}

Thema Kommunikation mit Patienten näher zu beschäftigen:

Der Anteil junger Patienten mit höherem Anspruch auf Informiertheit ist relativ hoch, und es gibt einen zunehmenden Informationsbedarf bei immer komplexeren und mehr technikbasierten diagnostischen und therapeutischen Verfahren. Hohe Implantatkosten können erforderliche Revisionen sehr kostspielig machen. Auf der anderen Seite werden die Zeitvorgaben für Gespräche durch Operationstätigkeit und zunehmende Knappheit von ärztlichem Personal immer enger.

Großen Einfluss hat auch gerade in der Orthopädie und Unfallchirurgie die leichte Dokumentierbarkeit von Fehlergebnissen, was sicherlich zum großen Teil zur führenden Rolle dieses Fachgebiets in den Behandlungsfehlerstatistiken beitrug. Nach der statistischen Erhebung der Gutachterkommissionen und Schlichtungsstellen für 2012 ist die Fachgebietsbeteiligung der Unfallchirurgie/Orthopädie etwa 10-mal so hoch wie die der Urologie (1999 vs. 197 von insgesamt 6212 Anträgen aus dem Krankenhausbereich). Unter den 10 häufigsten Einzeldiagnosen bei anerkanntem Behandlungsfehler im Krankenhausbereich findet sich nur eine (Cholelithiasis), die nicht das orthopädischunfallchirurgische Fachgebiet betrifft [8]. In keinem anderen Fach hat es also der Patient so leicht, den Vorwurf einer fehlerhaften Behandlung vorzubringen und auch zu belegen.

Auslöser von Behandlungsfehlervorwürfen sind nicht zuletzt immer wieder Kommunikationsmängel. Ein Behandlungsfehler muss nicht in einen Behandlungsfehlervorwurf münden. Was einen Fehler erst inakzeptabel macht, sind feh- lende Ehrlichkeit, Offenheit und Verantwortungsübernahme. Fehlender Blickkontakt, eine strenge Stimme und ein fehlendes Lächeln rufen Rachegefühle hervor, wenn die Behandlung nicht erfolgreich ist. Die Häufigkeit von Schadenersatzprozessen korreliert mit der Qualität der Kommunikation zwischen Arzt und Patient $[16,17]$. Medizinstudenten, die in Kommunikationstests schlecht abschneiden, sind im Rahmen ihrer späteren Tätigkeit als Ärzte sehr viel häufiger Adressaten von Patientenbeschwerden [26].

\section{Kommunikation im berufsgenos- senschaftlichen Heilverfahren}

Es stellt sich die Frage, welche Besonderheiten sich im BG-lichen (BG: Berufsgenossenschaft) Heilverfahren ergeben.

Eine Behandlung im BG-lichen Heilverfahren ist zwar primär der Unfallchirurgie zuzuordnen und unterscheidet sich zunächst nicht von der eines Nicht-BGPatienten. Das Aufgabenspektrum im BG-lichen Heilverfahren ist jedoch weiter gefasst und geht daher zwangsläufig auch mit höheren Ansprüchen an die Kommunikation einher [22, 24]. Zu diesen weitergehenden Aufgaben gehören:

- mit allen geeigneten Mitteln den durch den Arbeitsunfall verursachten Gesundheitsschaden zu beseitigen oder zu bessern, seine Verschlimmerung zu verhüten und seine Folgen zu mildern,

- dem Versicherten einen seinen Neigungen und Fähigkeiten entsprechenden Platz im Arbeitsleben zu sichern und

- Hilfen zur Bewältigung der Anforderungen des täglichen Lebens und zur
Teilhabe am Leben in der Gemeinschaft sowie zur Führung eines möglichst selbstständigen Lebens bereitzustellen.

Was die eigentliche unfallchirurgische Behandlung betrifft, sind die Rahmenbedingungen für eine erfolgreiche Kommunikation bei BG- und Nicht-BG-Patienten identisch (- Tab. 1). Dabei ist immer nach der Möglichkeit zu suchen, in einer erste Orientierung und Sicherheit vermittelnden Weise zu reagieren [14].

\section{Kommunikation bei Eintritt des Versicherungsfalls}

Anlässe, Orte und Termine einer Kommunikation zwischen Arzt und Patient in der Phase des Eintritts des Versicherungsfalls sind

- die Notfallbehandlung bzw. der Erstkontakt in der Sprechstunde,

- das präoperative Aufklärungsgespräch,

- die postoperative Visite,

- die täglichen Routinevisiten,

- die Chefarztvisiten und

- das Entlassungsgespräch.

Notfallbehandlung. Hier besteht häufig der größte Improvisationsbedarf: Essenziell sind die Vorstellung mit Name und Funktion sowie ein adäquater Blickkontakt. Der zeitliche, örtliche und inhaltliche Rahmen muss ansonsten der jeweiligen Situation angepasst werden. Wichtig ist, Störfaktoren nach Möglichkeit auszuschalten oder sie wenigstens anzukündigen. Bei Zeitnot sollte man um Verständnis bitten und auf einen geeigneteren Zeitpunkt, Rahmen oder auch Gesprächspartner verweisen.

Aufklärung. Die Aufklärung vor einer Operation ist Kommunikation in Reinform, hier liegt sogar die Beweislast beim Arzt. Dennoch gibt es auch hier vielfach noch Verbesserungspotenzial. Ein standardisierter Vordruck kann ein Aufklärungsgespräch nur ergänzen, nicht ersetzen. Die Informationsfülle ist gerade nach Unfällen für den Patienten verwirrend und beängstigend. Umso mehr muss sich der Arzt wiederholt fragen [25]: 
- Welche Informationen erreichen den Patienten und werden von ihm verstanden?

- Welche Informationen benötigt er, um sich kooperativ zu zeigen und um über gegebene Behandlungsalternativen zu entscheiden?

- Was muss bereits allein aus juristischen Gründen mitgeteilt werden?

- Wird ggf. die geistige und emotionale Aufnahmekapazität dadurch überfordert, dass die Informationen stark belastend sind?

- Nimmt der Patient Mitteilungen verzerrt auf?

- Ist er momentan überhaupt in der Lage, die vorgesehenen Informationen nachzuvollziehen?

Postoperative Kommunikation. Auch wenn sich der Operateur sicher ist, dass nach einer gelungenen Operation und einer genauen schriftlichen Anweisung zur postoperativen Behandlung alles bestens verlaufen ist und er sich nun wichtigeren Aufgaben widmen kann, sind postoperative Kontakte essenziell:

„Mit dem Erstkontakt, der Diagnose und einer noch so erfolgreich erscheinenden Frühphase der Behandlung ist die ArztPatient-Beziehung keineswegs beendet. Sie setzt sich fort in einer fachlichen und menschlichen Begleitung, die der Chirurg als Bezugsperson erkennen und wahrnehmen muss. Die Begegnung erfüllt sich also nur mit der weiteren Begleitung, sie darf durch organisatorische Einwirkungen, Arbeitszeitregelungen oder mangelnde ärztliche Einstellung nicht gefährdet werden" [15].

Der Patient liest sozusagen aus dem Gesicht des Arztes ab, wie eine Operation verlaufen und ob der Arzt mit seiner Arbeit zufrieden ist. Ist dies der Fall, kann auch der Patient zufrieden sein. Selbst der kürzeste Besuch signalisiert: „Ich kümmere mich noch um Sie und bin bereit $z u$ handeln, wenn etwas Unerwartetes eintreten sollte" und "Ich interessiere mich für Ihr Befinden, bin stolz auf meine Arbeit und freue mich, wenn es Ihnen von Tag zu Tag besser geht". Ein Patient, bei dem nach der Operation kaum ein Kontakt mit dem Arzt erfolgt, entwickelt schnell das Ge-

Trauma Berufskrankh 2014 · 16[Suppl 1]:16-20～DOI 10.1007/s10039-013-2028-4

(c) Springer-Verlag Berlin Heidelberg 2013

\section{P.-M. Hax \\ Kommunikation im berufsgenossenschaftlichen Heilverfahren. Besonderheiten der Arzt-Patienten-Kommunikation bei Eintritt des Versicherungsfalls}

\section{Zusammenfassung}

Bedeutung der Kommunikation zwischen Arzt und Patient. Eine gelungene Kommunikation hat in der Arzt-Patienten-Beziehung nachweislich für beide Seiten Vorteile. Der Arzt muss umdenken, sich auf den informierten Patienten einstellen und inn in eine Entscheidungsfindung einbeziehen. Gerade in Orthopädie und Unfallchirurgie sind nicht zuletzt Kommunikationsmängel immer wieder Auslöser von Behandlungsfehlervorwürfen, was die besondere Bedeutung der ArztPatient-Kommunikation in diesem Gebiet unterstreicht.

Besonderheiten im berufsgenossenschaftlichen Heilverfahren. Das im berufsgenossenschaftlichen Heilverfahren weiter gefasste Aufgabenspektrum geht zwangsläufig auch mit höheren Ansprüchen an die Kommunikation einher. Die hierfür erforderlichen Voraussetzungen schuf die gesetzliche Unfallversicherung durch die besondere Qualifikation, Ausstattung und Stellung des Durchgangsarztes (D-Arzt), insbesondere durch die Neuausrichtung der Heilverfahren in den letzten Jahren, die mit einer entsprechenden Verlagerung der Qualitätsanforderungen der DÄrzte und einer wachsenden Bedeutung von Kommunikationsaspekten im berufsgenossenschaftlichen Heilverfahren einhergeht.

\section{Schlüsselwörter}

Kommunikation · Berufsgenossenschaftliches Heilverfahren · D-Arzt · Patientensicherheit . Behandlungsfehler

\section{Communication in occupational insurance association healthcare. Characteristics of physician-patient communication in health insurance cases}

\begin{abstract}
Importance of communication between physician and patient. A successful communication in the physician-patient relationship has proven to have advantages for both parties. The physician must rethink, adapt to an informed patient and to include the patient in the decision making procedure. In orthopedics and trauma surgery in particular, communication deficits recurrently lead to accusations of treatment errors which underlines the special importance of physician-patient communication in this field.

Characteristics of occupational insurance association healthcare procedure. The extended spectrum of tasks included in the occupational insurance healthcare procedure is automatically associated with higher demands on communication. The requirements
\end{abstract}

fühl, ein beliebiges Rädchen im großen Krankenhausgetriebe zu sein oder - was ebenso tragisch ist - er schöpft Verdacht und schlussfolgert, dass irgendetwas nicht gut gelaufen ist und folglich verschwiegen werden muss [14]. for this were created by the statutory accident insurance through the special qualification, design and position of the occupational insurance consultant physician (D-Arzt), particularly with respect to the reorganization of the healthcare procedure in recent years. This has led to a corresponding shift in quality requirements on the D-Arzt and an increasing importance of communication aspects in the occupational insurance association healthcare procedure.

\section{Keywords}

Communication - Occupational insurance association healthcare - Accident insurance consultant physician $\cdot$ Patient safety $\cdot$ Medical errors 
ter und zieht sich einen Fersenbeinbruch zu. Nach einer längeren vorherigen Erwerbslosigkeit hatte er seit 4 Monaten wieder eine Anstellung. Er ist Alleinverdiener, hat 2 Kinder, seine Ehefrau hat keinen Führerschein, und er wurde zunächst in eine Nicht-VAV-Klinik (VAV: Verletzungsartenverfahren) eingeliefert. Neben den reinen Verletzungsfolgen müssen auch die Enttäuschung wegen des drohenden erneuten Verlusts des Arbeitsplatzes, die Wut wegen des Fremdverschuldens sowie die Sorgen um die Versorgung der Familie und wegen der anstehenden vorübergehenden Trennung bei Verlegung in eine wohnortferne Klinik berücksichtigt werden.

\section{Rahmenbedingungen}

D-Arzt (Durchgangsarzt). Die Voraussetzungen für eine umfassende, nicht nur die rein unfallchirurgischen Aspekte einer erlittenen Verletzung berücksichtigende Behandlung schuf die DGUV (Deutsche Gesetzliche Unfallversicherung) durch die Etablierung des D-Arztes. Dieser ist nach mindestens 8-jähriger Weiterbildung fachlich besonders qualifiziert und verfügt über eine standardisierte gehobene technische Ausstattung. Er hat außerdem einen besseren Zugriff auf erweiterte Diagnostik- und Therapiemöglichkeiten. Für ihn besteht eine Verpflichtung zur berufsbegleitenden durchgangsärztlichen Fortbildung, auch in den Bereichen Gutachtenwesen, Heilverfahren/Rehabilitationsmanagement und -medizin. Die Beherrschung der Systematik der Heilverfahren inklusive Formularwesen wird vorausgesetzt. Durch Mindestfallzahlen (250 pro Jahr im D-Arzt-Verfahren, 75 pro Jahr im VAV) soll ein hoher Qualitätsstandard gewährleistet werden [3, 6, 22].

Der D-Arzt ist eine wichtige Schaltstelle im BG-lichen Heilverfahren: Er ist nicht nur Erbringer medizinischer Leistungen, sondern als Betreuer des Verletzten und Repräsentant der Berufsgenossenschaft auch Erbringer von administrativen und Beratungsleistungen. Er ist die erste Kontaktperson bei ganzheitlicher Aufnahme des Verletzten in ein eng verzahntes Betreuungs- und Leistungssystem, legt fest, welchen weiteren Weg das jeweilige Heilverfahren nehmen soll und trägt gemein- sam mit der BG dafür Sorge, dass die Unfallverletzten von Anfang an eine besonders fachkundige Beratung und eine optimale Behandlung „mit allen geeigneten Mitteln“ erhalten. Im BG-lichen Heilverfahren ist also eine Kommunikation ohne frühzeitiges Einbeziehen der Verwaltung nicht denkbar. Der D-Arzt spielt hier eine wichtige Vermittlerrolle zwischen dieser und dem Verletzten. Über das Erbringen medizinischer Leistungen hinaus ist es seine Aufgabe, diese Dreiecksbeziehung aufzubauen, die anstelle der sonst üblichen Zweierbeziehung zwischen Arzt und Patient tritt. Er wird daher oft auch als Lotse im Heilverfahren bezeichnet.

Qualitätssicherung. Die Neuausrichtung der Heilverfahren in der DGUV sieht die Einrichtung eines Qualitätssicherungssystems vor, u. a. mit einem Fragebogen zur gesundheitsbezogenen Lebensqualität und Patientenzufriedenheit unter besonderer Berücksichtigung UV-relevanter (UV: Unfallversicherung) Versorgungsabläufe zur kontinuierlichen Überprüfung der Prozess- und Ergebnisqualität. Vor diesem Hintergrund ist es denkbar, dass es eine erfolgsorientierte Beteiligung von Krankenhäusern oder eine qualitätsorientiert befristete Zulassung geben wird. Dies führt zu einer Verlagerung der Qualitätsanforderungen an D-Ärzte - vom operierenden Unfallchirurgen zum Case-Manager der gesetzlichen Unfallversicherung $[1,5,11,20,21]$.

\section{Resümee}

Es muss auf jeden Fall davon ausgegangen werden, dass bei der bekannt positiven Wirkung der Kommunikation auf Lebensqualität und Patientenzufriedenheit die Bedeutung von Kommunikationsaspekten besonders im BG-lichen Heilverfahren wachsen wird. Besondere Kommunikationsaufgaben in diesem ergeben sich durch die Merkmale der besonderen Heilbehandlung, das Qualitätsmerkmal „Alles aus einer Hand“ und den Behandlungsauftrag „Mit allen geeigneten Mitteln“. Eine umfassende Betreuung durch D-Arzt, Sachbearbeiter und Rehabilitationsberater mit adäquater Hilfsmittelversorgung und beruflicher und sozialer Reintegration ist gerade vor dem Grundsatz „Rehabilitation vor Rente“ nur über eine effektive Kommunikation möglich. Unterstützend wirken dabei das etablierte strukturierte Berichtswesen und die Mitwirkungspflicht des Versicherten. Wird der Hinweis auf Letztere positiv gestaltet und entsprechend geschickt kommuniziert, lässt sich bereits dadurch ein erster Motivationsschub bewirken.

Eine besondere kommunikative $\mathrm{He}$ rausforderung für alle Beteiligten stellt die anstehende Verlegung in eine VAV-Klinik dar. Prestigedenken steht dem BG-lichen Auftrag entgegen und kann dem Heilerfolg nur schaden. Gefragt sind professionelle Argumentation und sachliche Information, sowohl des verlegenden Arztes als auch der aufnehmenden VAV-Klinik und der Verwaltung. Auch der Verletzte selbst kann hier im positiven Sinn mitwirken, wenngleich man ihn dahingehend nicht verpflichten kann.

\section{Fazit für die Praxis}

- Die Vorteile einer gelungenen Kommunikation sind heute wissenschaftlich erwiesen und dringen immer mehr ins Bewusstsein der Leistungserbringer und der Kostenträger im Gesundheitswesen.

- Die in vielen anderen Ländern bereits weit fortgeschrittene Integration einer entsprechenden Ausbildung in das Medizinstudium kommt auch in Deutschland allmählich in Gang.

- Betriebswirtschaftliche Überlegungen und Kosten-Nutzen-Analysen werden dazu führen, dass man der Verbesserung ärztlicher Kommunikationsfertigkeiten größere Aufmerksamkeit schenken wird. Keine Klinik wird es sich wirtschaftlich mehr leisten können, sich den Anforderungen an die interaktive und kommunikative Kompetenz ihrer Ärzte zu verschließen.

- Unter den Gesichtspunkten der Wirtschaftlichkeit, Rechtssicherheit, Personalbindung und unter Marketingaspekten wird eine umfassende Kommunikation immer wichtiger. Insbesondere die DGUV mit ihrem besonderen Behandlungsauftrag und ihrem hohen Qualitätsstandard sollte hier Maßstäbe setzen. 


\section{Korrespondenzadresse}

\section{Dr. P.-M. Hax}

Klinik für Orthopädie und Unfallchirurgie, BG-Unfallklinik Duisburg, Großenbaumer Allee 250, 47249 Duisburg hax@hax.de

\section{Einhaltung ethischer Richtlinien}

Interessenkonflikt. P.-M. Hax gibt an, dass kein Interessenkonflikt besteht.

Dieser Beitrag beinhaltet keine Studien an Menschen oder Tieren.

The supplement containing this article is not sponsored by industry.

\section{Literatur}

1. Andro KH (2010) Überlegungen zu Änderungen der ambulanten Versorgung. Trauma Berufskrankh [Suppl 1] 12:16-18

2. Armstrong J, Holland J (2004) Surviving the stresses of clinical oncology by improving communication. Oncology 18:363-368

3. Beickert R (2011) Anforderungen an den Durchgangsarzt - Aktueller Stand. Trauma Berufskrankh [Suppl 1] 13,:75-78

4. Böker W (2003) Arzt-Patient-Beziehung: Der fragmentierte Patient. Dtsch Arztebl 100(1-2):A-24/B22/C-22

5. Bönninghoff N (2010) Notwendige Veränderungen des berufsgenossenschaftlichen Heilverfahrens - Sicht des niedergelassenen Arztes. Trauma Berufskrankh [Suppl 1] 12:12-15

6. Bonnaire F (2012) Voraussetzungen der D-Arzt-Anerkennung in den neuen Heilverfahren. Trauma Berufskrankh [Suppl 2] 14:243-246

7. Brown SJ (1999) Patient-centered communication. Annu Rev Nurs Res 17:85-104

8. Bundesärztekammer (2012) Statistische Erhebung der Gutachterkommissionen und Schlichtungsstellen für das Statistikjahr. Bundesärztekammer, Berlin. http://www.bundesaerztekammer.de/downloads/Erhebung_StaeKo_mit_Zahlen_2012 komplett.pdf. Zugegriffen: 12.09.2013

9. Charles C, Gafni A, Whelan TJ (1999) Decision-making in the physician-patient encounter: revisiting the shared treatment decision-making model. Soc Sci Med 49:651-661

10. Edwards N, Kornacki MJ, Silversin J (2002) Unhappy doctors: what are the causes and what can be done? BMJ 324:835-838

11. Ernst $O$ (2010) Qualitätssicherung in der Heilbehandlung der gesetzlichen Unfallversicherung Messung der Ergebnisqualität im D-Arzt-Vefahren Diplomica, Hamburg, ISBN 978-3-8366-8716-4

12. Geisler L (2002) Arzt-Patient-Beziehung im Wandel - Stärkung des dialogischen Prinzips. In: Deutscher Bundestag (Hrsg) Schlussbericht der Enquete-Kommission Recht und Ethik der modernen Medizin. Springer VS, Wiesbaden, S 216-220

13. Gordon GH, Baker L, Levinson W (1995) Physicianpatient communication in managed care. West J Med 163:527-531
14. Hax PM (2011) Sicherheit durch Klarheit - Kommunikation in Unfallchirurgie und Orthopädie. In: Hax PM, Hax-Schoppenhorst T (Hrsg) Kommunikation mit Patienten in der Chirurgie - Praxisempfehlungen für Ärzte aller operativen Fächer. Kohlhammer, Stuttgart, ISBN 978-3-1702-1613-6

15. Hierholzer G, Hierholzer S (1989) Begegnung mit dem Patienten. In: Hierholzer G, Hierholzer S (Hrsg) Chirurgisches Handeln. Fragen - Überlegungen Antworten. Thieme, Stuttgart New York

16. Hobgood C, Hevia A, Tamayo-Sarver JH et al (2005) The influence oft he causes and contexts of medical errors on emergency medicine residents' responses to their errors: an exploration. Acad Med 80:758-764

17. Hochreutener MA (2008) Reden ist alles - Schweigen ist Folter - kurze Prozesse, aber richtig! Heilende Kommunikation nach Zwischenfällen. 18. Münchner Qualitätsforum, München

18. Langewitz W, Denz M, Keller A et al (2002) Spontaneous talking time at start of consultation in outpatient clinic: cohort study. BMJ 325:682-683

19. Maguire P, Pitceathly C (2002) Key communication skills and how to acquire them. BMJ 325:697-700

20. Oberscheven M (2010) Änderungen der stationären Versorgung - Überlegungen aus Sicht der DGUV. Trauma Berufskrankh [Suppl 1] 12:5-8

21. Oberscheven M (2011) Neuausrichtung der Heilverfahren. Trauma Berufskrankh [Suppl 1] 13:4547

22. Rybak C, Lenz O, Ehlers APF (2008) Das Berufsgenossenschaftliche Heilverfahren. Orthop Unfallchi Up2date 3:345-366

23. Schnack D (2012) Grüße aus Babel - Patienten verstehen Ärzte oft nicht. Ärzte Zeitung. http://www. aerztezeitung.de/praxis_wirtschaft/w_specials/ special-arzt-patient/article/822133/gruesse-babelpatienten-verstehen-aerzte-oft-nicht.html. Zugegriffen: 12.09.2013

24. Scholz AO, Schürmann J, Kortmann HR (2006) Berufsgenossenschaftliches Heilverfahren. Trauma Berufskrankh 8:257-264

25. Schweickhardt A, Fritzsche K (2009) Kursbuch ärztliche Kommunikation. Deutscher Ärzteverlag, Köln, ISBN 978-3-7691-3412-4

26. Tamblyn R, Abrahamowicz M, Dauphinee D et al (2007) Physician scores on a national clinical skills examination as predictors of complaints to medical regulatory authorities. JAMA 298:993-1001

27. Wilker FW (1994) Medizinische Psychologie und medizinische Soziologie, 2. Aufl. Urban \& Schwarzenberg, München Wien Baltimore, ISBN 978-35411-2492-3 